\title{
Green Synthesis of Highly Concentrated Aqueous Colloidal Solutions of Large Starch- Stabilised Silver Nanoplatelets
}

\author{
Fei Cheng, Jonathan W. Betts and Stephen M. Kelly* \\ Department of Chemistry, University of Hull, Cottingham Road, Hull, HU6 7RX, United \\ Kingdom
}

Andrew L. Hector

Chemistry, University of Southampton, Southampton, SO17 1BJ, United Kingdom

\begin{abstract}
A simple, environmentally friendly and cost-effective method has been developed to prepare a range of aqueous silver colloidal solutions, using ascorbic acid as a reducing agent, watersoluble starch as a combined crystallising, stabilising and solubilising agent, and water as the solvent. The diameter of silver nanoplatelets increases with higher concentrations of $\mathrm{AgNO}_{3}$ and starch. The silver nanoparticles are also more uniform in shape with the greater the diameter of the nanoparticles. Colloidal solutions with a very high concentration of large, flat, hexagonal silver nanoplatelets ( $230 \mathrm{~nm}$ in breadth) have been used to deposit and fix an antibacterial coating of these large starch-stabilised silver nanoplates on commercial cotton fibers, using a simple dip-coating process using water as the solvent, in order to study the dependence of the antibacterial properties of these nanoplatelets on their size.
\end{abstract}

*Corresponding author: Tel: +44 1482 466347; E-mail: s.m.kelly@hull.ac.uk

Keywords: silver, nanoplatelets, seed-mediated synthesis, aqueous colloidal solutions, antibacterial textiles, water-soluble starch, green chemistry

\section{Introduction}

Noble metal nanoparticles have attracted strong interest for their potential applications as catalytic, electronic, optical, environmental and biomedical materials. [1-5] Silver is one of the most studied noble metal elements due to its attractive physical properties and wide potential applications. [6-12] Various chemical methods have been developed to prepare silver nanoparticles with different sizes and shapes, such as spherical nanoparticles, nanobelts, nanowires, nanocubes and nanoplates. [13-26] However, these procedures suffer from a range of disadvantageous features: undesirable reagents, such as hydrazine hydrate, as the reducing agent; volatile organic chemicals as the solvent; surfactants and/or polymer stabilising agents; 
the production of powders or colloidal solutions with only relatively low concentrations of silver nanoparticles and, as a result, unsuitability for large-scale production.

Therefore, it is important to develop "green chemistry" approaches [27] to prepare silver nanoparticles of the desired size, shape, and aspect ratio as stable aqueous colloidal solutions with a high concentration for practical applications, such as in antibacterial coatings for bedding, wound dressings, hospital uniforms, etc. Generally, three aspects should be considered for a green method to prepare silver nanoparticles: environmentally friendly solvent medium and reducing agent and a non-toxic material for the stabilization of the nanoparticles. When water is used as the solvent, polysaccharides often serve as a reducing reagent or a capping reagent or, in some cases, as both a reducing and a capping reagent. [2832] Starch-capped silver nanoparticles with particle sizes below $25 \mathrm{~nm}$ have been prepared in aqueous solution by ultrasound-mediated, glucose reduction, [33] or normal thermal reduction [34, 35]. Khan et al. has reported the preparation of silver nanoparticles using ascorbic acid and starch as reducing and stabilizing agents, respectively. [36] Starch as a combined reducing and stabilizing agent has also been used to prepare silver nanoparticles $(10 \mathrm{~nm} \leq \mathrm{d} \leq 34 \mathrm{~nm})$ in an autoclave. [37]

Silver ions and silver compounds have been extensively utilised for their anti-septic and anti-microbial activity for more than 100 years, due to their toxic effect on some bacteria, viruses, algae and fungi.[4, 8, 38-39] Recently there has been much renewed interest in using silver as a broad-spectrum antimicrobial agent due to the increasing prevalence of antimicrobial resistant infections such as MRSA, especially in hospitals. Recent examples include the application of an antimicrobial gel containing silver nanoparticles in the size range of 7-20 nm in the treatment of burn and wound victims.[40] Several devices containing silver nanoparticles have recently been proposed for use in the fields of dental implantology, periodontology, and alveolar bone regeneration, for example, membranes for guided tissue regeneration (GTR) and guided bone regeneration (GBR) applications, scaffolds for bone regeneration, and dental implant coatings. [41]

Although small, spherical silver nanoparticles ( $4 \mathrm{~nm} \leq \mathrm{d} \leq 40 \mathrm{~nm}$ ) synthesised by green chemistry methods have been reported recently by us and other authors to show good antibacterial activity,[42-43] there appear to be no reports of the synthesis and evaluation of the antibacterial properties of large, anisotropic silver nanoparticles ( $d \geq 200 \mathrm{~nm}$ ), i.e., nanoplatelets, and of their deposition on substrates for practical applications. One of the main reasons for this lack of information is the fact that it is difficult to prepare relatively stable colloidal solutions of large size silver nanoparticles. In order to study the anti-microbial 
properties of large silver nanoplatelets, we have developed a novel green chemistry method, using ascorbic acid as reducing agent in water, for the preparation of highly concentrated, aqueous colloidal solutions of large, starch-stabilised, silver nanoplatelets with a controlled size, shape and aspect ratio. These aqueous colloidal solutions can then used in a very simple, but efficient, water-based, non-impact, dip-coating process, based on our previous work,[42] to deposit and fix a coating of silver nanoplates on the surface of cotton fibers, without the use of chemical binders, surfactants, dispersants, a post-deposition curing step, etc. [44] These highly concentrated solutions of silver nanoplatelets of a defined size, shape and aspect ratio could also facilitate the study of the quantum-confined physical properties of such silver nanoplatelets, such as surface plasmon resonance (SPR).

\section{Experimental}

\subsection{Materials}

Silver (I) nitrate $\left(\mathrm{AgNO}_{3}, 99+\%\right.$, product number 0696$)$ and sodium borohydride $\left(\mathrm{NaBH}_{4}\right.$, 96\%, product number 71321) were purchased from Lancaster and Fluka, respectively. Potato starch (product number S2004) and ascorbic acid (reagent grade, product number A7506) were sourced from Sigma-Aldrich. The cotton string fibers (diameter $1.2 \mathrm{~mm}$, product number: 719.504) are from Lyerco, Telford, UK. Ultrapure water with a specific resistance of 18.2 $\mathrm{M} \Omega \cdot \mathrm{cm}$ was obtained by reversed osmosis followed by ion-exchange and filtration (UPQ PS system, ELGA, USA).

\subsection{Synthesis of starch-protected silver seeds}

Seeds of starch-protected, silver nanoparticles were prepared by a procedure similar to that developed by Zou et al. [25] A 2.95 $\mathrm{M} \mathrm{AgNO}_{3}$ solution $\left(0.5 \mathrm{~cm}^{3}\right)$ was added to $0.5 \%$ aqueous starch solution $\left(99 \mathrm{~cm}^{3}\right)$. Then a $0.5 \mathrm{M} \mathrm{NaBH}_{4}$ solution $\left(1.0 \mathrm{~cm}^{3}\right)$, which had been aged for 2 $\mathrm{h}$, was added all at once. The resultant solution was stirred for $1 \mathrm{~h}$ and then aged for $24 \mathrm{~h}$ at room temperature to give a stable yellow silver colloidal solution $\left(\mathrm{S}_{0}\right)$. The solution exhibits a SPR band at $400 \mathrm{~nm}$ on the UV-vis spectrum. The silver seeds are nearly spherical with a diameter of $8.8 \mathrm{~nm} \pm 2.0 \mathrm{~nm}$ according to analysis using Transmission Electron Microscopy (TEM). 


\subsection{Stepwise seed-mediated synthesis of silver nanoparticles}

Method 1: Silver nanoparticles with different sizes and shapes were prepared using a stepwise, seed-mediated, growth strategy using ascorbic acid as a mild reducing agent in a dilute aqueous solution of soluble starch. Eight samples were prepared in total. A fresh $\mathrm{AgNO}_{3}-0.5 \%$ starch solution $(29.5 \mathrm{mM})$ was prepared by dissolving $\mathrm{AgNO}_{3}(0.50 \mathrm{~g})$ in an aqueous $0.5 \%$ starch solution $\left(100 \mathrm{~cm}^{3}\right)$. For the first reaction step, a portion of the $S_{0}$ silver nanoparticle seed solution $\left(5.0 \mathrm{~cm}^{3}\right)$ and a freshly prepared $1.0 \mathrm{M}$ ascorbic acid solution $(0.6$ $\mathrm{cm}^{3}$ ) were added to a $0.5 \%$ aqueous starch solution $\left(15 \mathrm{~cm}^{3}\right)$. A freshly prepared aqueous $\mathrm{AgNO}_{3}-0.5 \%$ starch solution $\left(10 \mathrm{~cm}^{3}\right)$ was then added dropwise to the resultant silverseed/starch/ascorbic acid reaction solution under strong stirring. A clear brown solution $\left(\mathrm{S}_{1}\right)$ was obtained after stirring at room temperature for $1 \mathrm{~h}$. For the following steps, $\mathrm{S}_{\mathrm{n}}$ samples were prepared by adding a portion of the $S_{\mathrm{n}-1}$ solution $\left(10 \mathrm{~cm}^{3}\right)$ and ascorbic acid solution $\left(0.6 \mathrm{~cm}^{3}\right)$ into a $0.5 \%$ starch solution $\left(10 \mathrm{~cm}^{3}\right)$ followed by dropwise adding of a freshly prepared $0.5 \% \mathrm{AgNO}_{3}$-starch solution $\left(10 \mathrm{~cm}^{3}\right)$. All the reactions were carried out by stirring at room temperature for $1 \mathrm{~h}$. Each of these experiments was repeated three times.

Method 2: A fresh $\mathrm{AgNO}_{3}-0.5 \%$ starch solution (29.5 mM) was prepared as described in method 1. For the first reaction step, a freshly prepared $1.0 \mathrm{M}$ ascorbic acid solution $\left(0.6 \mathrm{~cm}^{3}\right)$ was added to the $\mathrm{S}_{0}$ silver nanoparticle seed solution $\left(5.0 \mathrm{~cm}^{3}\right)$ and then a freshly prepared aqueous $\mathrm{AgNO}_{3}-0.5 \%$ starch solution $\left(10 \mathrm{~cm}^{3}\right)$ was added dropwise to the resultant silverseed/ascorbic acid reaction solution under strong stirring. A clear brown solution $\left(\mathrm{S}_{1}\right)$ was obtained after stirring at room temperature for $1 \mathrm{~h}$. For the following steps, $S_{n}$ samples were prepared by adding ascorbic acid solution $\left(0.6 \mathrm{~cm}^{3}\right)$ to a portion of the $\mathrm{S}_{\mathrm{n}-1}$ solution $\left(10 \mathrm{~cm}^{3}\right)$ followed by dropwise adding of a freshly prepared $0.5 \% \mathrm{AgNO}_{3}$-starch solution $\left(10 \mathrm{~cm}^{3}\right)$. All the reactions were carried out by stirring at room temperature for $1 \mathrm{~h}$. 


\subsection{Coating of silver nanoparticles on cotton fibers}

A thin film of silver nanoparticles was coated and fixed on the surface of cotton fibers (diameter: $1.2 \mathrm{~mm}$ ) by a simple immersion procedure, e.g., cotton fibers $(0.23 \mathrm{~g})$ were immersed in one of the $\mathrm{S}_{0}-\mathrm{S}_{8}$ colloidal solutions of silver nanoparticles $\left(15 \mathrm{~cm}^{3}\right)$, prepared according to the method described above, for 4 hours at room temperature. The resultant silver-coated fibers were then washed carefully with copious amounts of water, with no observable loss of silver nanoparticles, and then dried under vacuum overnight.

\subsection{Characterization methods}

UV-vis spectra were recorded in the range between 300 and $700 \mathrm{~nm}$ using a Perkin Elmer Lambda 25 spectrometer. The aqueous silver colloidal solutions, prepared as described above, were diluted by a factor of 100 so that their absorption could be measured in a quartz cuvette with a $1 \mathrm{~cm}$ optical path. Scanning electron microscopy (SEM) images were obtained using Carl Zeiss SMT 'EVO60' SEM operating at $20 \mathrm{kV}$ and EDX data were obtained using an Oxford Instruments 'INCA' Energy Dispersive X-ray Spectrometer. TEM images were collected using a Jeol 2010 TEM instrument running at $200 \mathrm{kV}$. Images were recorded using a Gatan Ultrascan 4000 digital camera. The liquid sample for TEM analysis was mixed well in a vial and then a $5 \mu \mathrm{L}$ aliquot was placed on a hydrophilic carbon coated copper grid and allowed to dry in air. Fourier Transform Infrared spectroscopy (FT-IR) for silver powder samples were recorded on a Nicolet Magna-500 FTIR spectrometer. X-ray powder diffraction (XRD) analyses were either performed using a SIEMENS D5000 instrument for analysing silver powder samples or a Bruker AXS D8 Discover with GADDS detector for analysing silver-coated cotton fibers. The silver powder samples for FTIR, XRD and SEM were obtained by centrifugation (Sigma Laborzentrifugen 2-15 Howe in combination with Fisher oak ridge $28 \mathrm{~mL}$ centrifuge tubes) at $10000 \mathrm{rpm}$ for $15 \mathrm{~min}$, washing (x3) with water and then drying under vacuum overnight. The concentration of the silver nanoparticles present on the surface of the fibers was determined using an inductively coupled Perkin Elmer plasma 40 emission ICP instrument. The solutions for ICP were prepared by dissolving the samples (100 $\mathrm{mg}$ ) in $3 \mathrm{ml}$ concentrated nitric acid (Romil Ltd, Cambridge UK, SpA grade) then heated to $200{ }^{\circ} \mathrm{C}$ in a sealed Teflon digestion vessel (CEM Xpress vessels) and, when cool, they were diluted to $15.0 \mathrm{~g}$. 


\subsection{Antimicrobial testing}

The antimicrobial activity of the silver-nanoparticle-coated cotton fibers was determined using the diffusion method against isolates of meticillin-resistant Staphylococcus aureus (MRSA) (NCTC 12493) and extended-spectrum $\beta$-lactamase (ESBL) producing Escherichia coli (NCTC 11560), purchased from Pro-lab Diagnostics, Wirral, UK. Acinetobacter baumannii (NCTC 12156) and Stenotrophomonas maltophilia (NCTC 10258) were purchased from the Health Protection Agency laboratories, Porton Down, UK. These bacterial isolates were chosen due to their high levels of antibiotic resistance and potentially life-threatening infections in a hospital and community environment. Individual plates of IsoSensitest agar (Oxoid, Basingstoke, UK) were inoculated with each of the bacterial isolates, using the standardized method by Moosden et al., (1988). [45] Samples of cotton fibers were added to the surface of the agar of each plate using sterile forceps. The agar plates were then incubated at $37{ }^{\circ} \mathrm{C}$ for 24 hours, with the exception of those inoculated with S. maltophilia, which were incubated at $30{ }^{\circ} \mathrm{C}$ for 24 hours. Six replicates were performed for each agar plate produced in order to confirm any apparent results. Zones of inhibition, i.e., the absence of bacterial growth surrounding the fibers, was then measured and recorded. Microtitre assays were performed in order to determine the antimicrobial performance of each colloidal solution of cellulose-stabilized silver nanoparticles in a liquid culture and also to determine their minimum inhibitory concentrations against specific bacterial isolates. The concentration of each of the $\mathrm{S}_{0}-\mathrm{S}_{8}$ cellulose-stabilized nanoparticle colloidal solutions was adjusted to give $1000 \mu \mathrm{g} / \mathrm{cm}^{-3}$ stock solutions. A double dilution from these stock solutions was performed a total of nine times into sterile, distilled water to give ten different concentrated solutions for each silver nanoparticle size. A sample $(100 \mu \mathrm{L})$ of each of these solutions of $\mathrm{S}_{0}$ silver nanoparticles was then pipetted into 10 horizontal wells of a 96 -well cell-culture plate (1 well per concentration) and sterile distilled water $(100 \mu \mathrm{L})$ was added as a control to well 11 . This process was repeated for each of the $\mathrm{S}_{1}-\mathrm{S}_{8}$ aqueous colloidal solutions of silver nanoparticles. An isolate of MRSA was pipetted into each well as a $100 \mu \mathrm{L}$ volume of a 0.5 MacFarland suspension in IsoSensitest broth. The entire procedure was repeated using E. coli, A. baumannii or S. maltophilia as the bacterial inoculant. Microtitre plates were incubated in a rocking incubator at $37{ }^{\circ} \mathrm{C}$ for 24 hours with the exception of those inoculated with $S$. maltophilia that were incubated at $30{ }^{\circ} \mathrm{C}$ for 24 hours. MICs were taken as the lowest concentrations not showing any visible growth. Six repeat experiments were conducted for each organism in order to confirm the validity of each result. 


\section{Results and discussion}

\subsection{Preparation of starch-stabilised, silver-nanoparticle colloidal solutions}

Ascorbic acid was used as a reducing agent to facilitate the formation of the silver nanoparticles and starch was used as a capping agent to stabilise them during and after formation. Ascorbic acid $\left(\mathrm{C}_{6} \mathrm{H}_{8} \mathrm{O}_{6}\right)$ is a mild reducing agent, but its redox potential is low enough to reduce silver ions to metallic silver, [46-47] according to the following equation:

$$
\mathrm{C}_{6} \mathrm{H}_{8} \mathrm{O}_{6}+2 \mathrm{Ag}^{+} \longrightarrow 2 \mathrm{Ag}^{0}+\mathrm{C}_{6} \mathrm{H}_{6} \mathrm{O}_{6}+2 \mathrm{H}^{+}
$$

When ascorbic acid is added drop-wise to solutions containing the silver seeds $S_{n-1}$, prepared in the previous step, and fresh silver ions, a significant colour change can be observed, especially in the first four steps. Figure 1 shows each of the silver colloidal solutions, $S_{1}-S_{8}$, prepared in each of the eight steps and the initial seed sample solution, $S_{0}$. The samples $S_{1}-S_{4}$ exhibit colours ranging from brown to red to purple due to their SPR absorption. Sample $\mathrm{S}_{6}$ exhibits a magnolia colour, whereas the samples $S_{7}$ and $S_{8}$ are colourless, turbid solutions. Sample $S_{0}$ is stable for more than three weeks. Very small amounts of precipitates can be observed for the solutions $\mathrm{S}_{1}-\mathrm{S}_{4}$ after two days, which are then stable thereafter, whereas the colloidal solutions $S_{5}$ and $S_{6}$ are still stable up to 12 hours, and $S_{7}$ and $S_{8}$ to four hours, when a small amount of precipitation can be observed. The $\mathrm{S}_{1}-\mathrm{S}_{8}$ samples can be returned to clear colloidal solutions again upon light shaking by hand and these regenerated solutions are then stable for similar times to those of the fresh solutions.

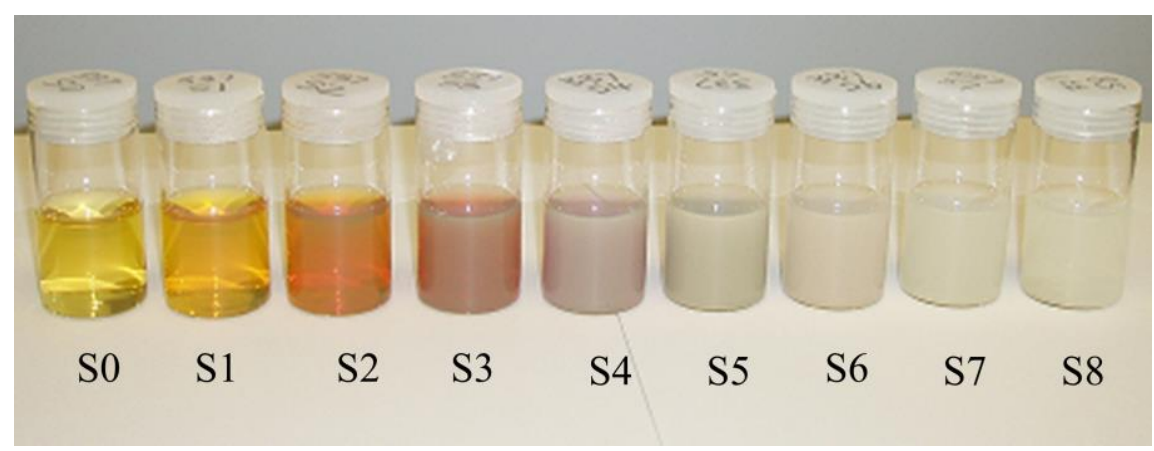

Figure 1 Photograph of colloidal solutions of silver nanoparticles prepared in a series of consecutive steps. A fresh $0.5 \%$ aqueous starch solution was added at each step. Each of the solutions has been diluted 50 times to facilitate analysis. 

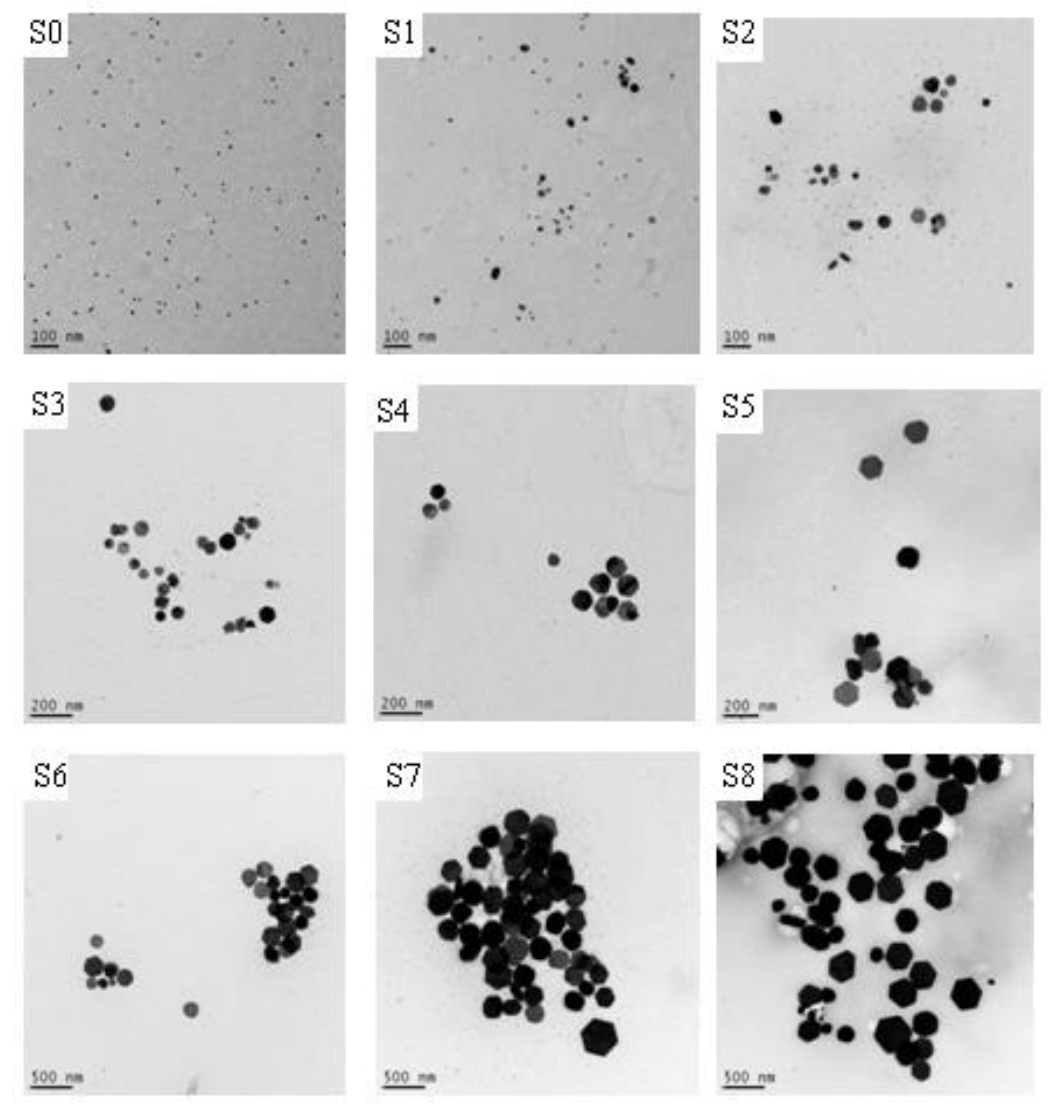

Figure 2 TEM images of the solid products obtained from the starch-stabilised silver nanoparticle colloidal solutions $\mathrm{S}_{0}-\mathrm{S}_{8}$.

Table 1 Summary of the results for starch-stabilised silver nanoparticles.

\begin{tabular}{|l|l|l|}
\hline Sample & $\begin{array}{l}\text { Approximate silver } \\
\text { nanoparticle size }(\mathrm{nm})\end{array}$ & Morphology \\
\hline & & \\
\hline $\mathrm{S}_{1}$ & $8-24$ & $\begin{array}{l}\text { some nanoparticles are similar to those of } \mathrm{S}_{0} \text { and } \\
\text { some have irregular shapes }\end{array}$ \\
\hline $\mathrm{S}_{2}$ & $15-45$ & $\begin{array}{l}\text { hexagonal nanoplates, nanorods and } \\
\text { irregularly shaped nanoparticles (majority) }\end{array}$ \\
\hline $\mathrm{S}_{3}$ & $25-60$ & many hexagonal nanoplates \\
\hline $\mathrm{S}_{4}$ & $47-73$ & hexagonal nanoplates (majority) \\
\hline $\mathrm{S}_{5}$ & $68-114$ & hexagonal nanoplates (majority) \\
\hline $\mathrm{S}_{6}$ & $83-190$ & hexagonal nanoplates \\
\hline $\mathrm{S}_{7}$ & $136-340$ & hexagonal nanoplates \\
\hline $\mathrm{S}_{8}$ & $148-467$ & hexagonal plates (majority at $\sim 230 \mathrm{~nm})$ \\
\hline
\end{tabular}

The TEM images of the samples $\mathrm{S}_{1}-\mathrm{S}_{8}$, as well as the seed sample $\mathrm{S}_{0}$, are shown in Figure 2 . The results obtained from analysis of these images are summarized in Table 1 for the starchstabilised silver nanoparticles present in each of the aqueous colloidal solutions. It can be 
seen that the silver nanoparticles are more uniform in shape for nanoparticles with a large diameter. The majority of nanoparticles in $S_{1}$ are similar to those observed in $S_{0}$, although some have grown to $24 \mathrm{~nm}$ in diameter. Some nanorods and hexagonal nanoplates have been formed in $S_{2}$, but the majority of nanoparticles exhibit an irregular shape. The amount of hexagonal nanoplates increases with the increase in the size of the nanoparticles present in the samples from $S_{3}$ to $S_{5}$. Finally, nearly all the nanoparticles in samples $S_{6}-S_{8}$ are hexagonal nanoplates. Most of the hexagonal plates in sample $\mathrm{S}_{8}$ are $c a .230 \mathrm{~nm}$ in breadth, although the nanoparticle sizes range from 148 to $467 \mathrm{~nm}$ at low concentration at the extremes of this range.

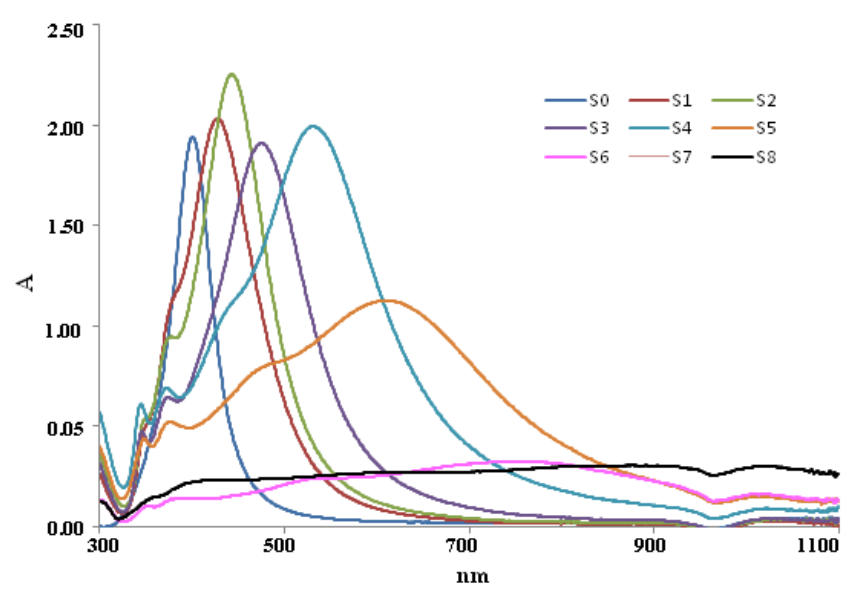

Figure 3 UV-vis absorption spectra of the starch-stabilised silver nanoparticle colloidal solutions prepared in the different steps $\mathrm{S}_{1}-\mathrm{S}_{8}$.

Figure 3 shows the UV-vis absorption spectra of the starch-stabilised silver nanoparticle colloidal solutions prepared in the nine different steps in the process. It can be seen that there is an absorption peak centred at $400 \mathrm{~nm}$ in the UV-vis absorption spectrum of the silver nanoparticle seed solution $\mathrm{S}_{0}$, indicating the presence of spherical silver nanoparticles. After the first reaction step, $S_{1}$, the main peak shifts to $428 \mathrm{~nm}$, accompanied by shoulders at 378 and $345 \mathrm{~nm}$, suggesting the formation of polygonal structures of silver nanoparticles. [48] The solutions $S_{2}$ and $S_{3}$ exhibit strong peaks at $465 \mathrm{~nm}$ and $498 \mathrm{~nm}$, respectively, accompanied by two much weaker absorption intensities at about $378 \mathrm{~nm}$ and $346 \mathrm{~nm}$. A strong peak at 539 $\mathrm{nm}$ accompanied by one shoulder at about $443 \mathrm{~nm}$ and two weak peaks (373 and $347 \mathrm{~nm}$ ) can be observed for solution $\mathrm{S}_{4}$. The presence of two or more surface plasmon resonance bands 
(SPR) is due to the formation of anisotropic, rather than spherical, nanoparticles. The frequency and intensity of the SPR absorption bands are mainly related to their diameter, size-distribution, the shape of the nanostructures, volume concentration (aggregated or isolated assembly) and the environment surrounding them.[49]

Omission of the $0.5 \%$ starch solution from the reactions above results in silver colloidal solutions with similar colour changes. However, in this case it takes twelve steps to produce a colourless, turbid solution. The TEM image of this colourless solution shows that the silver nanoparticles are not hexagonal nanoplates and that the nanoparticle size distribution is very broad (Figure 4).

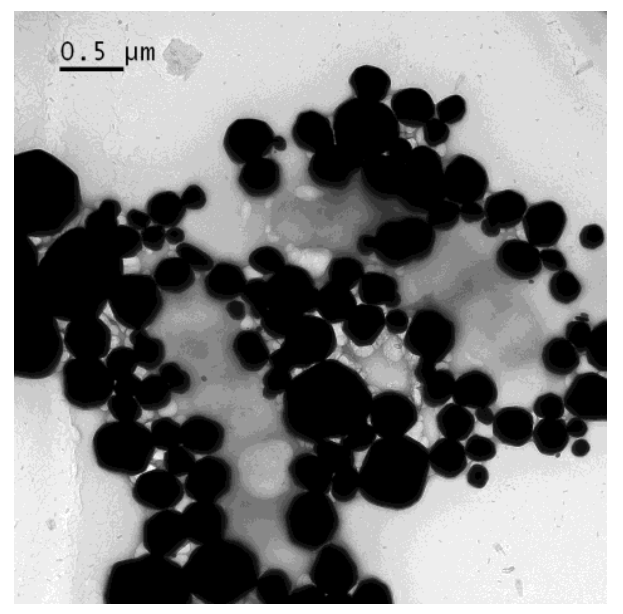

Figure 4 TEM image of the colourless aqueous colloidal solution of silver nanoparticles prepared by a method in which fresh $0.5 \%$ starch solution was not added in each step.

The exact mechanism for the growth of hexagonal nanoplates in this system is yet to be fully understood. The preparation of silver nanoplates, using sodium citrate, cetyl trimethylammonium bromide and glycyl glycine as capping agents, has been reported.[22, 25, 50-51] It has also been demonstrated that these capping agents play a crucial role in the twodimensional growth of silver nanoplates. Since the $\{111\}$ lattice plane of face-centered cubic silver may possess the lowest surface energy, the adsorption of these chemical species to this plane may further reduce the surface energy and, as a result, the nanoplates grow with $\{111\}$ as a basal plane. Suber et al. also reported that slow addition of ascorbic acid solution to a silver nitrate solution in the presence of Daxad 19 as capping agent led to the formation of hexagonal tabular anisotropic particles.[52] In our system, starch is the only capping agent present in the reaction solution. Although there appears to be no report of the adsorption abilities of starch on the different silver crystal faces, the evolution process of the silver structures from spherical nanoparticles into hexagonal nanoplates, as shown in Figure 2, 
suggests that the nanoplates may grow using $\{111\}$ as a basal plane. The weak peaks from 2810 to $3000 \mathrm{~cm}^{-1}$ in the IR spectra of the S6, S7 and S8 powders, which were obtained after centrifuging separation from the corresponding colloidal solutions, confirmed the attachment of starch on the silver particles surfaces (Figure 5). The hydrophilic poly-OH groups were mainly responsible for the adsorption of starch onto the surface of silver nanoparticles through electrostatic interactions.[36, 37] However, these hexagonal nanoplatelets formed in the aqueous starch solution disappear after removal of water by centrifugation and drying under vacuum, as shown in the SEM image in Figure 6. The powder XRD pattern of sample $\mathrm{S}_{8}$ also shows no preferred orientation (Figure 7), despite the fact that the data was collected in flat plate (Bragg-Brentano) geometry. The sample S6 and S7 have very similar XRD spectra to that of S8 (not shown), however no XRD spectra can be recorded for the samples from S0 to S5 because no corresponding powders could be obtained from the colloidal solutions by centrifugation. Larger nanoplates would have been expected to lie flat during sample preparation, so this result also suggests that they do not survive the dehydration and drying process. However, the nano-platelets are preserved intact, if the silver nanoparticles are directly coated on the substrate using the aqueous colloidal solutions, as shown in the TEM images.

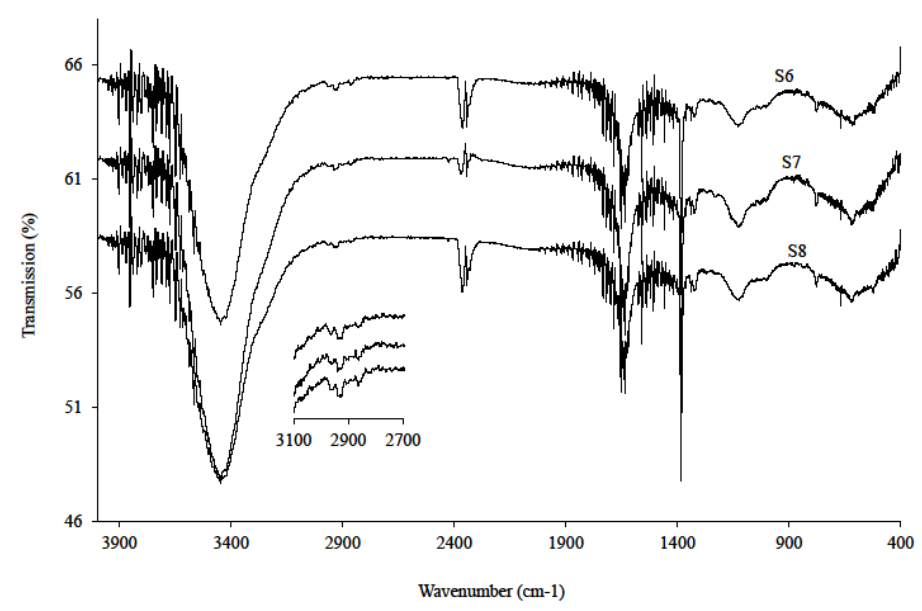

Figure 5 IR spectra of the starch-stabilised silver nanoparticle powder S6, S7 and S8 after removal of the water from the corresponding solutions and drying under vacuum. 


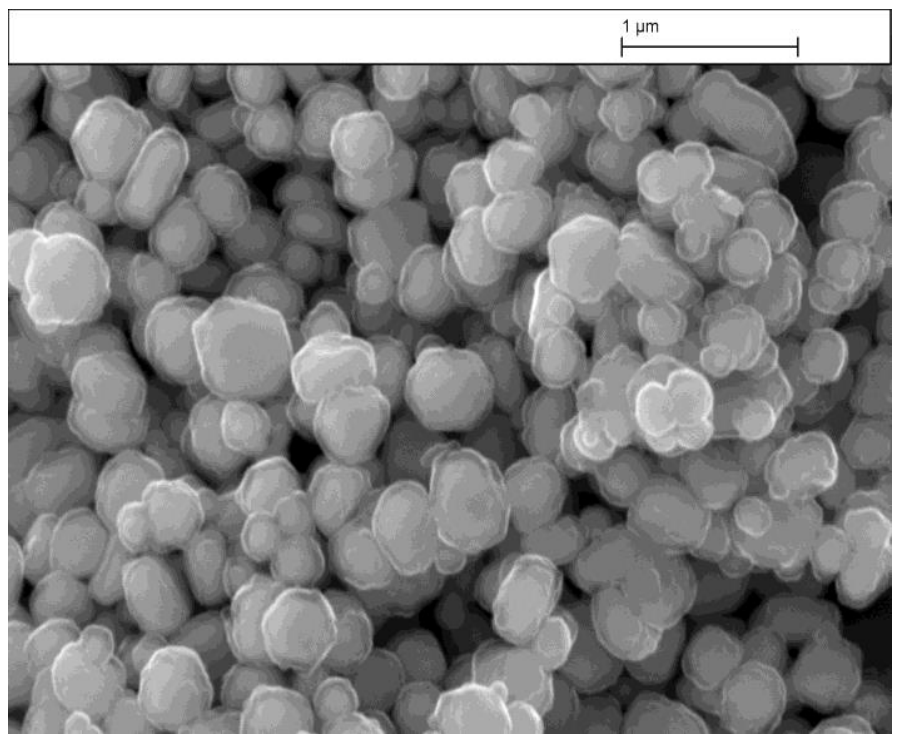

Figure 6 SEM image of the starch-stabilised silver nanoparticle powder after removal of the water from the $S_{8}$ solution and drying under vacuum.

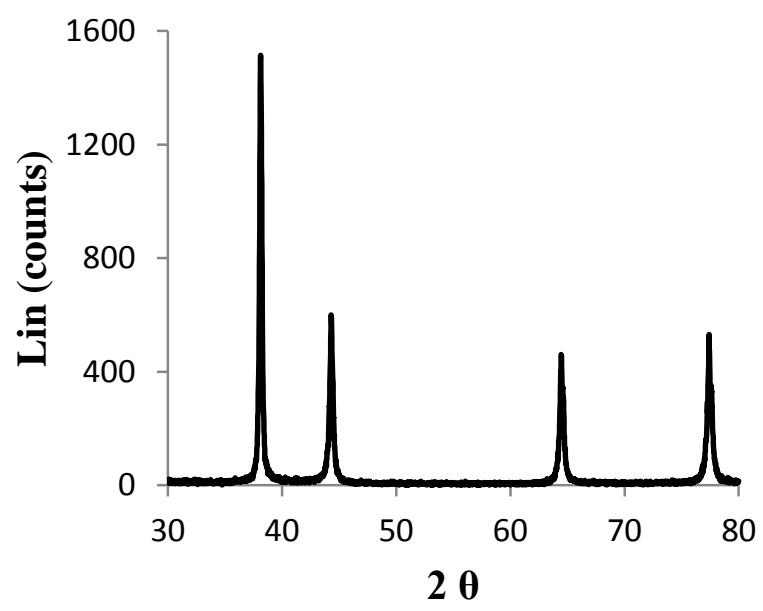

Figure 7 XRD pattern of the starch-stabilised silver nanoparticle powder $\mathrm{S}_{8}$ after drying under vacuum.

\subsection{Surface coating of fibres with silver nanoparticles}

The cotton fibers coated by a simple immersion method using each of the $\mathrm{S}_{0}-\mathrm{S}_{8}$ solutions exhibit different colours corresponding to the original colours of the silver nanoparticle colloidal solutions. The results of ICP analysis of the fibers $\mathrm{SF}_{\mathrm{n}}(\mathrm{n}=0-8)$ coated using the corresponding silver nanopartcle colloidal solutions, $S_{n}$, are shown in Table 2. Generally, the 
concentration of silver on dried silver-coated cotton fibers increases gradually from $0.37 \%$ on $\mathrm{SF}_{1}$ to $0.66 \%$ on $\mathrm{SF}_{8}$. The nature and amount of the coatings of silver nanoparticles on the cotton fiber surface were also confirmed by using XRD analysis, see Figure 8. It can be seen that the relative intensity of the peak at $2 \theta=37.68^{\circ}$ to peak at $2 \theta=45.01^{\circ}$ is higher for the fibers coated from the $S_{8}$ solution than the fibers coated from the $S_{2}$ solution. The preferred intensive peak at $2 \theta=37.68^{\circ}$ corresponds to the $\{111\}$ lattice plane of face-centered cubic silver, suggesting that the silver nanoplatelets from the $\mathrm{S}_{8}$ solution have been preserved after being coated on the cotton fibers.[25] The SEM images of the cotton fibers and silver-coated cotton fibers, shown in Figure 9, reveal that thin coatings of individual silver nanoparticles are present on the fiber surfaces, although some aggregates of a number of the silver nanoparticles can be also observed on the surfaces.

Table 2 The precentage of silver nanoparticles present on the $\mathrm{SF}_{0}-\mathrm{SF}_{8}$ cotton fibers coated using the corresponding colloidal solutions $\mathrm{S}_{0}-\mathrm{S}_{8}$.

\begin{tabular}{llllllllll}
\hline Fibres & $\mathrm{SF}_{0}$ & $\mathrm{SF}_{1}$ & $\mathrm{SF}_{2}$ & $\mathrm{SF}_{3}$ & $\mathrm{SF}_{4}$ & $\mathrm{SF}_{5}$ & $\mathrm{SF}_{6}$ & $\mathrm{SF}_{7}$ & $\mathrm{SF}_{8}$ \\
\hline $\mathrm{Ag}(\%)$ & 0.142 & 0.369 & 0.372 & 0.384 & 0.341 & 0.460 & 0.578 & 0.516 & 0.663 \\
\hline
\end{tabular}

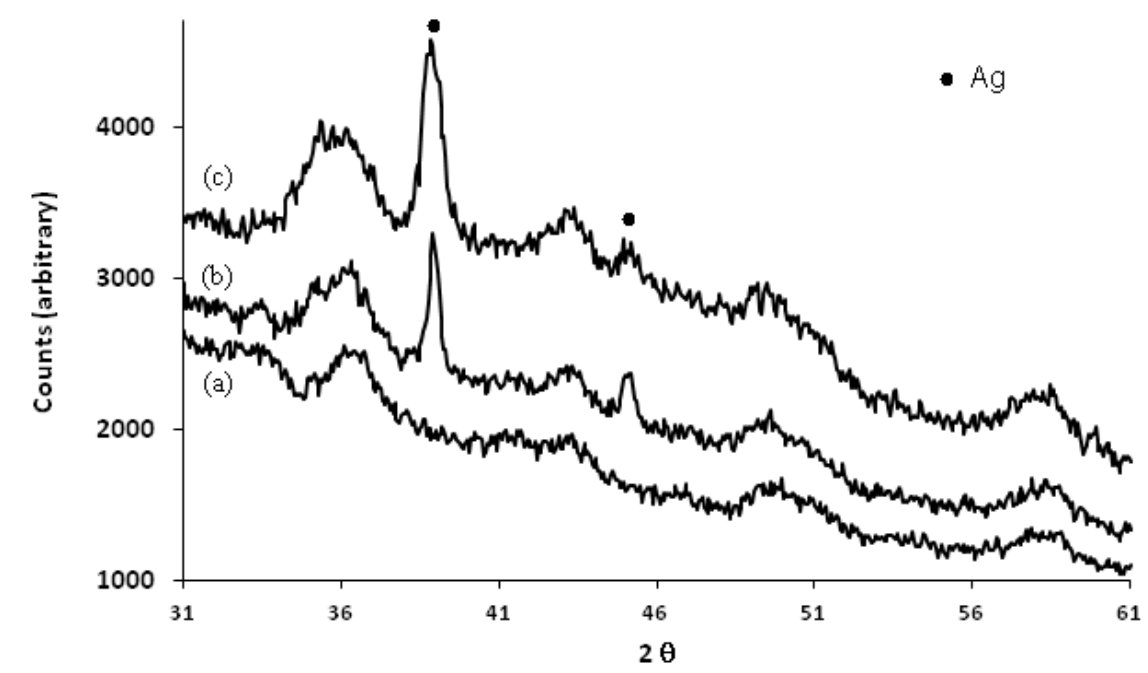

Figure 8 XRD patterns of silver-coated cotton fibers. (a) untreated cotton fibers, (b) silvernanoparticle-coated cotton fibers using the $S_{2}$ solution and (c) silver-nanoparticle-coated cotton fibers using the $\mathrm{S}_{8}$ solutions. 

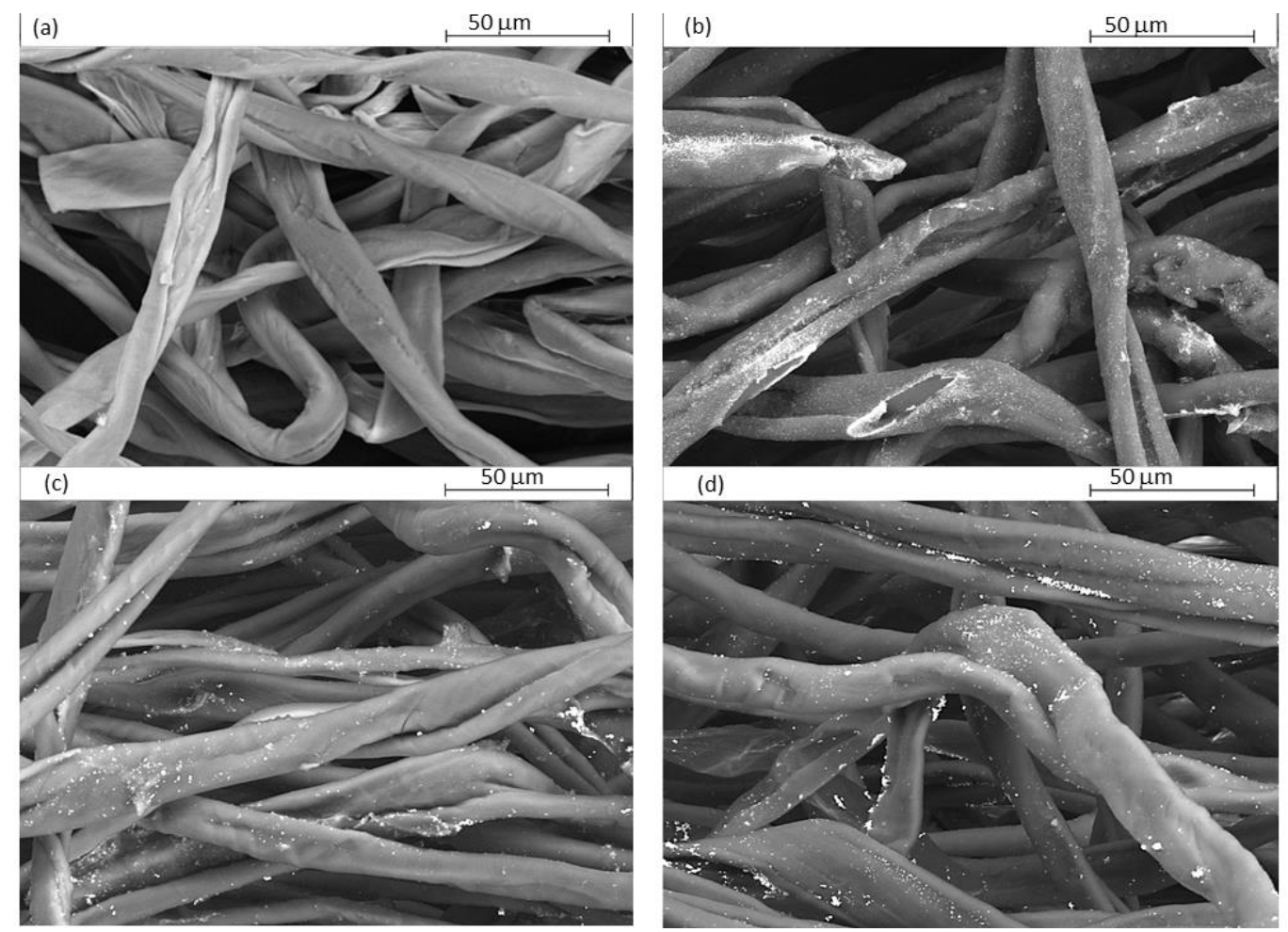

Figure 9 SEM images of cotton fibers and silver-coated cotton fibers: (a) untreated cotton fibers (b) $\mathrm{SF}_{2}$, (c) $\mathrm{SF}_{6}$ and (d) $\mathrm{SF}_{8}$ cotton fibers coated with silver nanoparticles using the corresponding aqueous colloidal solutions of silver nanoparticles, $S_{2}, S_{6}$ and $S_{8}$, respectively.

\subsection{Antimicrobial effects of starch-stabilised silver nanoparticles}

The antibacterial performance of the silver-coated, cotton fibers against S. maltophilia, E. coli, A. baumannii and MRSA was tested using a diffusion assay. Cotton fibers produced by soaking in the $\mathrm{S}_{0}-\mathrm{S}_{4}$ silver aqueous colloidal solutions created zones of inhibition against isolates of S. maltophilia, E. coli, A. baumannii and MRSA, indicating good antibacterial activity. The strongest antibacterial activity was observed with $\mathrm{SF}_{0}$ (Figure 10) where a zone of $9 \mathrm{~mm}$ (excluding width of the fiber) was produced. No zones of inhibition were observed around the corresponding $\mathrm{SF}_{5}-\mathrm{SF}_{8}$ cotton fibers coated using the $\mathrm{S}_{5}-\mathrm{S}_{8}$ aqueous colloidal solutions incorporating larger silver nanoplates, against any bacterial isolate. However, no bacterial growth was observed on or under the fibers suggesting antibacterial activity was still produced. 


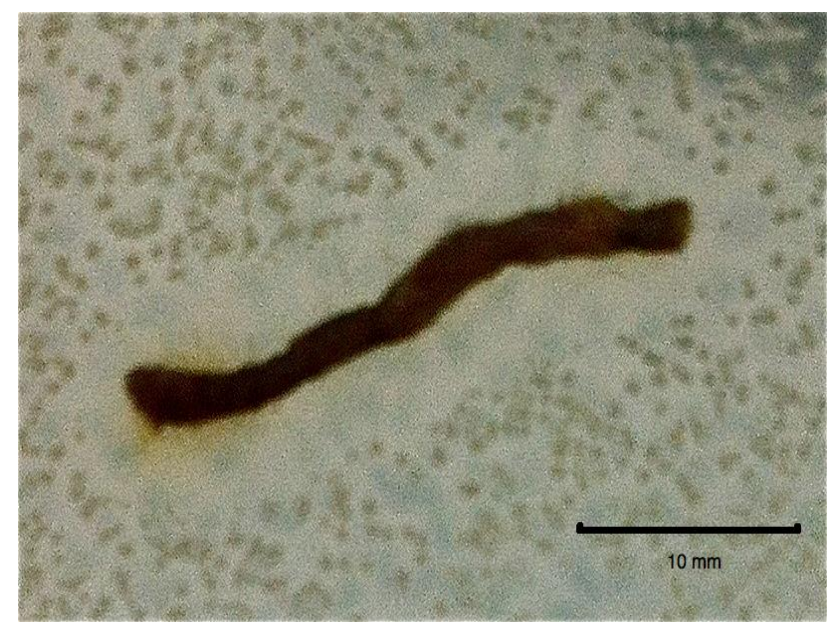

Figure 10 Antibacterial effect of the cotton fiber coated using the starch-stabilised silver nanoparticles present in the aqueous colloidal solution $\mathrm{S}_{0}$ against MRSA, represented by a zone of bacterial growth inhibition (no growth) surrounding the fiber.

The results from the microtitre assay tests show a high level of antibacterial activity for the aqueous colloidal solutions containing the $\mathrm{S}_{0}-\mathrm{S}_{6}$ silver nanoparticles against MRSA with minimum inhibitory concentrations (MIC) of $7.81 \mu \mathrm{g} \mathrm{cm}^{-3}$ determined for the $\mathrm{S}_{0}$ nanosphere colloidal solution, increasing to MIC values of up to $500 \mu \mathrm{g} \mathrm{cm}^{-3}$, when using $\mathrm{S}_{6}$ colloidal solution of nanoplates, see Table 3. No antibacterial action was observed when using the $S_{7}$ silver colloidal solution of nanoplates against MRSA. However, antibacterial action was observed using the $\mathrm{S}_{8}$ aqueous silver nanoplatelet colloidal solution giving a minimum inhibitory concentration of $250 \mu \mathrm{g} \mathrm{cm}^{-3}$, which is half the MIC value observed using the corresponding $\mathrm{S}_{5}$ and $\mathrm{S}_{6}$ colloidal solutions of silver nanoplatelets.

All the silver nanoparticle colloidal solutions $\left(\mathrm{S}_{0}-\mathrm{S}_{8}\right)$ show antibacterial action, when used against A. baumannii and $S$. maltophilia. The $\mathrm{S}_{0}$ colloidal solution of spherical silver nanoparticles produces the lowest MIC value $\left(7.81 \mu \mathrm{g} \mathrm{cm}^{-3}\right)$. The MIC values increase with increasing nanoparticle size, as well as a change in shape to flat, hexagonal nanoplates, up to a maximum value observed for the $S_{8}$ colloidal solution of the largest silver nanoplates, where the MIC value drops to $\leq$ half the MIC value observed for the $\mathrm{S}_{7}$ colloidal solution of slightly smaller nanoplates. E. coli appears to be the most resistant to silver nanoparticles sizes $S_{2}$ and above with no antibacterial action observed when using $S_{6}-S_{8}$ aqueous colloidal solutions of silver nanoplates. 
The difference between the antimicrobial activity observed for the silver nanoparticle colloidal solutions $\left(\mathrm{S}_{0}-\mathrm{S}_{8}\right)$, in the presence of either E. coli or MRSA, could be due to the differences in the cell walls of these two different types of bacteria.[8] However, the difference in antibacterial action observed for the aqueous silver nanoparticle colloidal solutions $\left(\mathrm{S}_{0}-\mathrm{S}_{8}\right)$, in the presence of either E. coli, A. baumannii or S. maltophilia, is not likely be attributable to differences in their cell walls, as all three bacterial species are gram negative. The difference in activity could, perhaps, be due to other differences in their cell physiology, such as variance of porins, or differences in their mechanisms of active efflux.

Table 3 Minimum inhibitory concentrations of stabilized silver nanoparticles against bacterial isolates $\left(\mu \mathrm{g} \mathrm{cm}^{-3}\right)$.

\section{Bacterial isolate}

\begin{tabular}{ccccc} 
Ag nanoparticle & MRSA & E. coli & A. baumannii & S. maltophilia \\
\cline { 2 - 5 } & 7.81 & 7.81 & 7.81 & 7.81 \\
$\mathrm{~S}_{0}$ & 31.25 & 125 & 125 & 62.5 \\
$\mathrm{~S}_{1}$ & 62.5 & 250 & 125 & 125 \\
$\mathrm{~S}_{2}$ & 125 & 250 & 125 & 125 \\
$\mathrm{~S}_{3}$ & 250 & 500 & 250 & 250 \\
$\mathrm{~S}_{4}$ & 500 & 500 & 500 & 250 \\
$\mathrm{~S}_{5}$ & 500 & N/A & 500 & 250 \\
$\mathrm{~S}_{6}$ & N/A & N/A & 500 & 250 \\
$\mathrm{~S}_{7}$ & 250 & N/A & 250 & 62.5 \\
$\mathrm{~S}_{8}$ & & &
\end{tabular}

N/A - Not applicable, MIC not reached. All MIC values are expressed as a mean of 6 individual results.

The antibacterial activity of the starch-stabilised $\mathrm{S}_{0}$ silver nanoparticles observed in this report is at least as good as that reported in the literature using different methods of synthesis.[37, 53] The antibacterial action of the $S_{1}-S_{2}$ silver nanoparticles produced in this research is also comparable to previous work, where galactose was used as the stabilising agent.[5] The poorer antibacterial action of the larger silver nanoplates, especially against the gram negative isolates, is probably be due to a lower surface area-to-volume ratio of 
nanoplates compared to analogous nanospheres and especially compared to the very small nanospheres, see Table 1, present in the seed nanoparticle solution, $S_{0}$. The relatively small surface area of the larger nanoplates should lead to a lower diffusion of silver ions, responsible for the antibacterial activity of silver nanoparticles, from the larger nanoplates. Another possible reason is reduction in silver permeability through bacterial cell wall/cell membranes, due to the larger size of the nanoplates, and a consequent blocking of their mode of action. However, this explanation is not consistent with the higher antibacterial action of $\mathrm{S}_{8}$ silver nanoparticles, which is seen in all bacterial isolates with the exception of E. coli. However, a possible, alternative explanation for this difference in activity could be that the larger silver nanoplates present in the $S_{8}$ colloidal solution may break into smaller nanoparticles, while diffusing through the bacterial cell wall/cell membranes, taking into account the fact that these large nanoplates are easily broken, as discussed above, see Figures 6 and 7. However, further investigation is required to determine the exact mechanism behind the higher degree of activity observed for the $\mathrm{S}_{8}$ solution compared to the activity for the colloidal solutions $\mathrm{S}_{5}-\mathrm{S}_{7}$ containing smaller silver nanoplates, see table 2 . We believe this is the first occasion where large stable Ag NPs have been shown to produce antibacterial activity against multidrug-resistant bacteria such as S. maltophilia, an opportunistic hospital pathogen.

\section{Conclusions}

A novel, environmentally friendly, sustainable method has been developed for the synthesis of large, starch-stabilised, silver nanoplatelets, which uses water-soluble starch, in the presence of ascorbic acid as a reducing agent of silver nitrate, to influence the shape, size and aspect ratio of the silver nanoplatelets, as well as acting as a crystallising, stabilising and solubilising agent. The presence of the starch is crucial to the preparation of a series of stable, aqueous colloidal solutions containing large, flat, hexagonal silver nanoplates, whose size depends on the number of sequential seeded reaction steps. These highly concentrated, aqueous colloidal solutions can be used to deposit and fix a coating of starch-stabilised silver nanoparticles on cotton fibers, using a simple dip-coating process in water at room temperature, with a pronounced antibacterial activity against antimicrobial resistant bacteria, such as S. maltophilia and MRSA. 


\section{Acknowledgements}

The research leading to these results has received funding from the European Union Seventh Framework Programme (FP7/2007-2013) under the Grant Agreement Number 214653 as the SURFUNCELL project. Mrs A Lowry, Mr A Sinclair and Mr R Knight are thanked for providing TEM, SEM and ICP analyses, respectively. 


\section{References}

[1] V. K. Sharma, R. A. Yngard, Y. Lin, Adv. Colloid Interface Sci. 145 (2009) 83.

[2] K. D. Hermanson, S. O. Lumsdon, J. P. Williams, E. W. Kaler, O. D. Velev, Science 294 (2001) 1082.

[3] Z. H. Zhou, S. L. Wang, W. J. Zhou, G. X. Wang, L. H. Jiang, W. Z. Li, S. Q. Song, J. G. Liu, G. Q. Sun, Q. Xin, Chem. Commun. (2003) 394.

[4] J. S. Kim, E. Kuk, K. N. Yu, J-H. Kim, S. J. Park, H. J. Lee, S. H. Kim, Y. K. Park, Y. H. Park, C-Y. Hwang, Y-K. Kim, Y-S. Lee, D. H. Jeong, M-H. Cho, Nanomedicine:NBM 3 (2007) 95.

[5] A. Panacek,L. Kvitek,R. Prucek, M. Kolar, R. Vecerova, N. Pizurova, V. K. Sharma,. T. Nevecna, R. Zboril, J. Phys. Chem. B 110 (2006) 16248.

[6] P.C. Lee, D. Meisel, J. Phys. Chem. 86 (1982) 3391.

[7] A. I. Lukman, B. Gong, C. E. Marjo, U. Roessner, A. T. Harris, J. Colloid Interf. Sci, 353 (2011) 433.

[8] T. Maneerung, S. Tokur, R. Rujiravanit, Carbohyd. Polym. 72 (2008) 43.

[9] M. Maillard, S. Giorgio, M. P. Pileni, J. Phys. Chem. B 107 (2003) 2466.

[10] G. McNay, D. Eustace, W. E. Smith, K. Faulds, D. Graham, Appl. Spectrosc. 65 (2011) 825.

[11] N. R. Jana, L. Gearheart, C. J. Murphy, Chem. Commun. (2001) 617.

[12] J. J. Mock, M. Barbic, D. R. Smith, D. A. Schultz, S. Schultz, J. Chem. Phys. 116 (2002) 6755.

[13] N. Shirtcliffe, U. Nickel, S. Schneider, J. Colloid Interf. Sci. 211 (1999) 122.

[14] S. Schneider, P. Halbig, H. Grau, U. Nickel, Photochem. Photobiol. 60 (1994) 605.

[15] Y.G. Sun, B. Mayers, Y.N. Xia, Nano Lett. 3 (2003) 675.

[16] B. Wiley, Y.G. Sun, Y.N. Xia, Accounts Chem. Res. 40 (2007) 1067.

[17] I. Washio, Y. Xiong, Y. Yin, Y. Xia, Adv. Mater. 18 (2006) 1745.

[18] B. Wiley, Y. G. Sun, B. Mayers, Y. N. Xia, Chem.-Euro. J. 11 (2005) 454.

[19] Y. Xia, Y. Xiong, B. Lim, S. E. Skrabalak, Angew. Chem. Int. Edit. 48 (2009) 60.

[20] I. Pastoriza-Santos, L.M. Liz-Marzan, J. Mater. Chem. 18 (2008) 1724.

[21] S. H. Chen, D. L. Carroll, Nano Lett. 2 (2002) 1003. 
[22] J. Yang, L. Lu, H. Wang, W. Shi, H. Zhang, Cryst. Growth Des. 6 (2006) 2155.

[23] Y. G. Sun, Y. N. Xia, Adv. Mater. 15 (2003) 695.

[24] Y. Xiong, A. R. Siekkinen, J. Wang, Y. Yin, M. J. Kim, Y. Xia, J. Mater. Chem. 17 (2007) 2600.

[25] X. Zou, E. Ying, H. Chen, S. Dong, Colloid Surface A 303 (2007) 226.

[26] I. Sondi, D. V.Goia, E. Matijevic, J. Colloid Interf. Sci., 260 (2003) 75.

[27] P. Raveendran, J. Fu, S. L. Wallen, J. Am. Chem. Soc. 125 (2003) 13940.

[28] P. Raveendran, J. Fu, S. L. Wallen, Green Chem. 8 (2006) 34.

[29] D. K. Bozanic, L. V Trandafilovic, A. S. Luyt, V. Djokovic, React. Funct. Polym. 70 (2010) 869 .

[30] E. Filippo, A. Serra, A. Buccolieri, D. Manno, J. Non-Crystal. Solids 356 (2010) 344.

[31] H. Z. Huang, X. R. Yang, Carbohyd. Res. 339 (2004) 2627.

[32] M. N. Nadagouda, R. S. Varma, Biomacromolecules 8 (2007) 2762.

[33] P. Vasileva, B. Donkova, I. Karadjova, C. Dushkin, Colloid Surface A 382 (2011)

203.

[34] D. Manno, E. Filippo, M. Di Giulio, A. Serra, J. Non-Crystal. Solids 354 (2008) 5515.

[35] X. Gao, L. Wei, H. Yan, B. Xu, Mater. Lett. 65 (2011) 2963.

[36] Z. Khan, T. Singh, J. I. Hussain, A. Y. Obaid, S. A. AL-Thabaitib, E.H. ElMossalamyb, Colloid Surface B 102 (2013) 578.

[37] N. Vigneshwaran, R. P. Nachane, R. H. Balasubramanya, P. V. Varadarajan, Carbohyd. Res. 341 (2006) 2012.

[38] M. Liong, B. France, K. A. Bradley, J. I. Zink, Adv. Mater. 21 (2009) 1684.

[39] P. Totaro, M. Rambaldini, Interact. Cardiovasc. Thorac. Surg. 8 (2009) 153.

[40] J. Jain, S. Arora, J. M. Rajwade, P. Omray, S. Khandelwal, K. M. Paknikar, Mol. Pharmaceut. 6 (2009) 1388.

[41] S. Sivolella, E. Stellini, G. Brunello, C. Gardin, L. Ferroni, E. Bressan, B. Zavan, J. Nanomater. (2012) 12.

[42] F. Cheng, J. W. Betts, S. M. Kelly, J. Schaller, T. Heinze, Green Chem. 15 (2013) 
989.

[43] D. Breitwieser, M. M. Moghaddam, S. Spirck, M. Baghbanzadeh, T. Pivec, H.

Fasl, V. Ribitsch, C. O. Kappa, Carbohyd. Polym. 94 (2013) 677.

[44] M. Pollini, M. Russo, A. Licciulli, A. Sannino, A. Maffezzoli, J. Mater. Sci.Mater. M. 20 (2009) 2361.

[45] F. Moosdeen, J. D. Williams, A. Secker, J. Antimicrob. Chemoth. 21 (1988) 439.

[46] D.V. Goia, E. Matijevic, New J. Chem. 22 (1998) 1203.

[47] K. P. Velikov, G. E. Zegers, A. van Blaaderen, Langmuir 19 (2003) 1384.

[48] L. P. Jiang, S. Xu, J. M. Zhu, J. R. Zhang, J. J. Zhu, H. Y. Chen, Inorg. Chem. 43 (2004) 5877.

[49] E. Hutter, J. H. Fendler, Adv. Mater. 16 (2004) 1685.

[50] M. Maillard, P. R. Huang, L. Brus, Nano Lett. 3 (2003) 1611.

[51] S.H. Chen, Z. Y. Fan, D .L. Carroll, J. Phys. Chem. B 106 (2002) 10777.

[52] L. Suber, I. Sondi, E. Matijevic, D. V. Goia, J. Colloid Interf. Sci., 288 (2005) 489.

[53] L. He, S-Y. Gao, H. Wu, X-P. Liao, Q. He, B. Shi, Mater. Sci. Eng. C 32 (2012) 1050 . 